\title{
Quantized Multiskyrmions as Possible Nuclear Bound States of Antikaons
}

\author{
Vladimir Kopeliovich* \\ Institute for Nuclear Research of Russian Academy of Sciences, Moscow 117312, Russia \\ E-mail: kopelioeinr.ru

\section{Irina Potashnikova} \\ Universidad Técnica Federico Santa María, Casilla 110-V, Valparaíso, Chile \\ E-mail: irina.potashnikova@utfsm.cl
}

\begin{abstract}
The chiral soliton aproach (CSA) provides a conceptually new way to understanding the nature of many body bound states, which is in complete correspondence with experiments. The spectrum of baryonic states is calculated as a function of external quantum numbers (baryon number, strangeness, isospin etc.), but their particular hadronic content is not fixed. Just same situation takes place in, e.g., missing mass experiments where the spectrum of states is measured at fixed values of baryon number, strangeness etc., but internal hadronic content cannot be defined, in principle.

There is a rigorous statement within the CSA that the number of states below the antikaon nucleus threshold increases with decreasing kaon mass. At physical values of the kaon mass and other model parameters several states below the antikaon - nucleus threshold are predicted within the CSA. These states could overlap and appear in experiment as broad enhancement seen by FINUDA and recently by DISTO Collaboration.
\end{abstract}

Xth Quark Confinement and the Hadron Spectrum

8 - 12 October 2012

TUM Campus Garching, Munich, Germany

\footnotetext{
* Speaker.

${ }^{\dagger}$ Based on papers V.Kopeliovich, I.Potashnikova, Phys.Rev. C83, 064302 (2011); V.Kopeliovich, B.Schwesinger, B.Stern, Nucl. Phys. A549, 485 (1992).
} 
1. Recent intensive discussion [1] - [6] of the possible antikaon - nuclei bound states proposed first in [1] emphasized once more the absence of the complete relativistic many-body theory of bound states. In the standard approach the hadronic content of the composed state is fixed at the first steps, at next steps one tries to solve corresponding (usually nonrelativistic) equations. One of the problems of relativistic generalizations is that the amount of constituents of composed states is not fixed, and cannot be fixed, in principle. For example, in the case of the $B=2, S=-1$ state it could be hyperon-nucleon, antikaon-nucleon-nucleon, hyperon-nucleon-pion, hyperon-nucleontwo-pion, etc., components of the Fock column. So called meson assisted dibaryons have been discussed recently in [7].

The chiral soliton approach (CSA), or topological Skyrme model, opens a conceptually new way to understand the nature and to describe the spectrum of multibaryon states, i.e. the states with baryon number $B>1$. The CSA is based on few ingredients and basic principles incorporated in the model lagrangian (see [6] for the list of references). The baryon number is the degree of the map $R^{3} \rightarrow S^{3}$ of our 3-dimensional space to the manyfold of the chiral fields (configuration space, see e.g. references in [6]).

2. The advantage of the CSA is that multibaryon states - nuclei, hypernuclei ... - can be considered on equal footing with $\mathrm{B}=1$ case. The rational map approximation [8] simplifies this work considerably and allows to calculate easily such characteristics of classical configurations as moments of inertia, sigma term etc., which enter the quantization procedure.

It is useful to ascribe ordinary nuclei and hypernuclei (ground states) to definite $S U(3)$ multiplets, see fig. 1 below for the $B=2$ case. In a version of the bound state quantization model it is possible to describe total binding energies of light hypernuclei in qualitative, even semiquantitative agreement with data [9]. Some kind of the "bag model" is obtained with universal properties of the bag envelope, starting with the effective lagrangian [10].

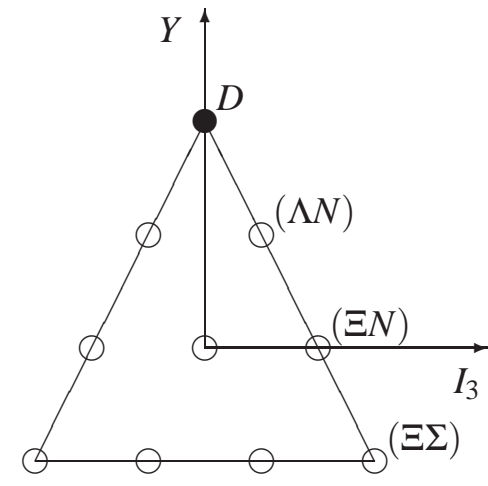

$B=2,\{\overline{10}\}, J=1$

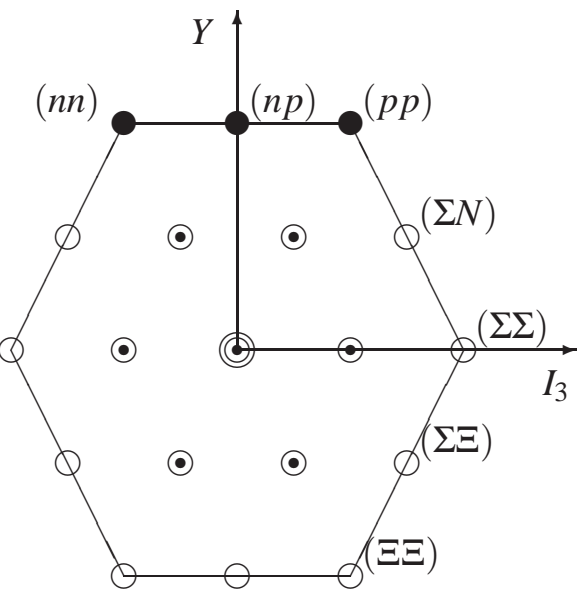

$B=2,\{27\}, J=0$

Fig 1 . The $I_{3}-Y$ diagrams of multiplets of dibaryons, $B=2$. The nonstrange components, the deuteron $(J=1)$ and the $N N$ scattering state $(J=0)$ are indicated by black circles. Virtual levels with nonzero strangeness are indicated in brackets, e.g. $(\Lambda N)$ scattering state which appears as a near threshold enhancement of the $\Lambda N$ interaction cross section [11]. 
Certain rotational excitations of bound skyrmions can be interpreted as deeply bound $\bar{K}$ nuclear states [6].

3. Within CSA a rigorous statement can be made: in the limit of small $m_{K}$ there are bound states below the antikaon-nucleus threshold, and the number of such states increases with decreasing_value of the kaon mass $m_{K}$ [6]. For realistic value of $m_{K}$ there are at least few bound states with positive and negative parities, different values of spin $J$ and isospin $I$ (fig. 2).

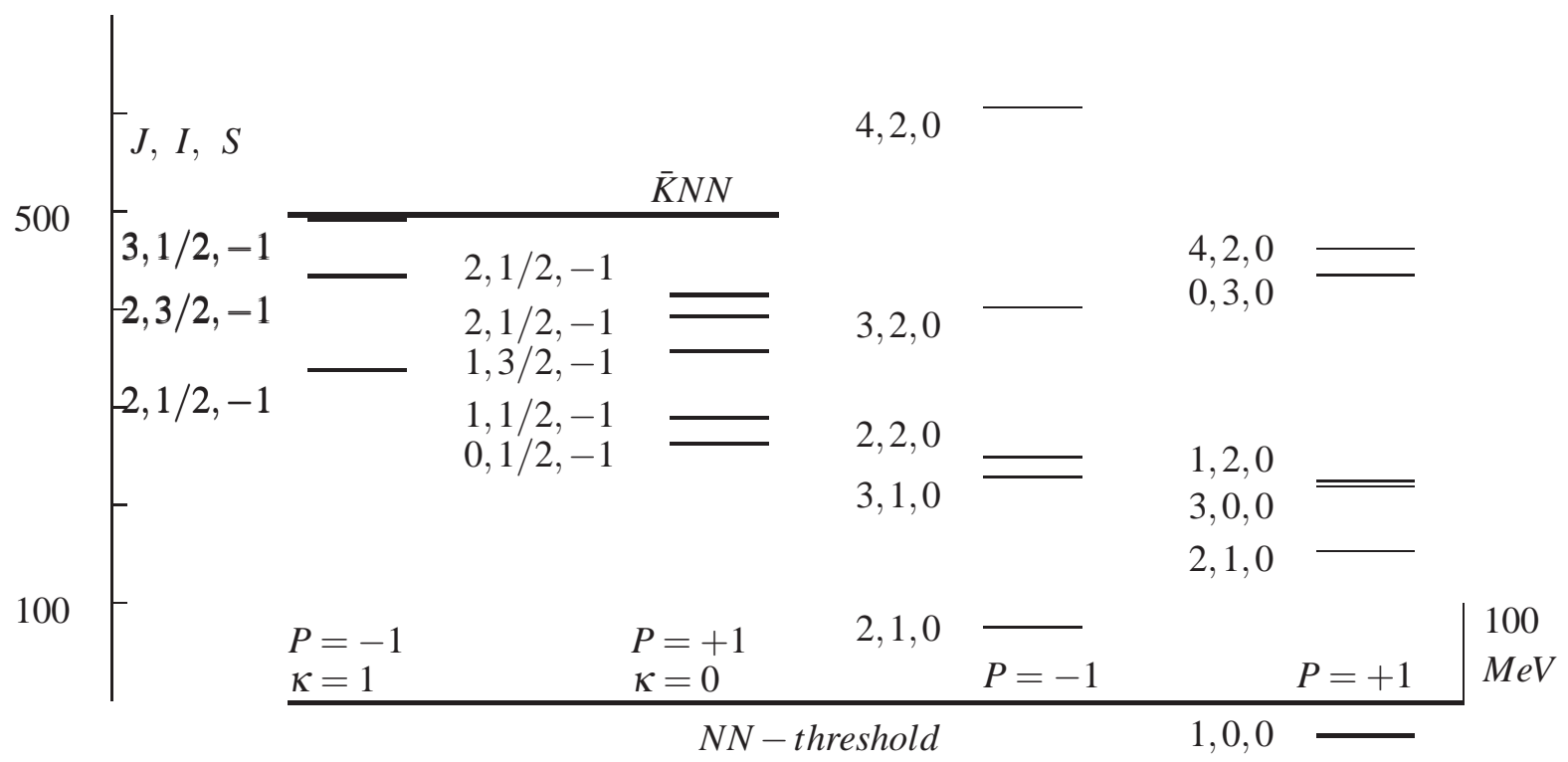

Fig. 2. Position of the $B=2$ states (above the $N N$ threshold) with negative strangeness, negative and positive parities (first 2 columns); with zero strangeness, negative and positive parities (columns 3 and 4). The $\bar{K} N N$ threshold is shown by black line, as well as the $N N$ threshold. Accuracy of this calculation is not better than $\sim 40 \mathrm{MeV}$ (figure from [6]).

Specifics of the basic $B=2$ toroidal configuration $[12,13]$ is that the integer $\kappa$ (3-d projection of the body-fixed isospin of quantized state) defines the parity of quantized states, see [13]. Rotational excitations have additional energy $\Delta E=J(J+1) /\left(2 \Theta_{J}\right)$. The lowest $S=-1$ states with $I=1 / 2, J^{P}=2^{-}, I=1 / 2, J^{P}=0^{+}$and $J^{P}=1^{+}$have greatest chances to be interpreted as $\bar{K} N N$ compound state with binding energy $\sim 100 \mathrm{Mev}$.

The orbital inertia grows fast with increasing baryon (atomic) number, $\Theta_{J} \sim B^{p}, p$ is between 1 and 2, see e.g. [9]. By this reason the number of rotational states becomes greater for large baryon numbers. Some of them can be interpreted as deeply bound antikaon-nuclei states, proposed in [1]. The particular case of the $B=3$ system has been partially investigated in [6]. More detailed investigations are necessary, especially for the $B>2$ multibaryons.

Besides the collective rotational excitations there are also vibration-breathing excitations considered in [14] for nonstrange dibaryons and not investigated still for strange multibaryons.

4. Within the CSA several $S=-1$ states below the antikaon-nucleus threshold have been predicted with various values of isospin and spin [6]. The explicit hadronic content of these states cannot be fixed in CSA. These states could overlap in the missing mass experiments and appear 
as broad enhancements of corresponding cross sections, seen by FINUDA [15] and DISTO [16] collaborations in the $B=2$ systems. So, the "incomplete information" about the nature of such states provided by the CSA may be, however, sufficient for description of existing data. An actual experimental task is to resolve the states with different values of spin and isospin.

The work has been supported by Fondecyt (Chile), grant numbers 1090236 and 1130549.

\section{References}

[1] Y. Akaishi and T. Yamazaki, Nucl. Phys. A684, 409 (2001); Phys. Rev. C65, 044005 (2002)

[2] T. Yamazaki and Y. Akaishi, Phys. Rev. C76:045201 (2007); Y. Akaishi, A. Dote and T. Yamazaki, Phys. Lett. B613, 140 (2005)

[3] Y. Akaishi, K.S. Myint and T. Yamazaki, nucl-th/0805.4382

[4] E. Oset and H. Toki, nucl-th/0509048; V.K. Magas, E. Oset, A. Ramos and H. Toki, nucl-th/0601013

[5] L. Tolos, A. Ramos and E. Oset, Phys.Rev. C74, 015203 (2006)

[6] V. Kopeliovich and I. Potashnikova, Phys.Rev. C83, 064302 (2011)

[7] A. Gal, arXiv:1011.6322 [nucl-th]

[8] C. Houghton, N. Manton, P. Suttcliffe, Nucl. Phys. B510, 507 (1998)

[9] V. Kopeliovich, JETP, 96, 782 (2002)

[10] V. Kopeliovich, J.Phys. G28, 103 (2002)

[11] V. Kopeliovich, B. Schwesinger, B.Stern, Nucl. Phys. A549, 485 (1992)

[12] V. Kopeliovich and B. Stern, JETP Lett. 45, 88 (1987)

[13] E. Braaten and L. Carson, Phys. Rev. D38, 3525 (1988)

[14] B. Schwesinger and N. Scoccola, Phys. Lett. B363, 29 (1995)

[15] M. Agnello et al (FINUDA Collab.) Phys.Rev.Lett. 94, 212303 (2005)

[16] M. Maggiora et al (DISTO Collab.) Nucl. Phys. A835, 43 (2010) 